Ann. Zootech., I970, 19 (4), 423-437.

\title{
INFLUENCE DE LA FERTILISATION AZOTÉE SUR LA VALEUR ALIMENTAIRE DES FOURRAGES VERTS
}

\author{
C. DEMARQUILLY \\ avec la collaboration technique de J.-M. Borssau, H. Bousguet et G. Cuylde \\ Station de Recherches sur l'Élevage des Ruminants, \\ Centre de Recherches de Clermont-Ferrand, \\ 63 -Saint-Genès-Champanelle \\ Institut national de la Recherche agronomique
}

\section{RÉSUMÉ}

Io Nous avons comparé en continu tout au long des cycles successifs de végétation l'influence de la fertilisation azotée sur la digestibilité de l'herbe verte et la quantité qui en est ingérée par les moutons.

L'étude a porté sur :

- une prairie de ray-grass anglais Melle Pâtuve ayant reçu après le premier cycle 5o ou Ioo kg $\mathrm{N} /$ ha. Le deuxième cycle a été étudié pendant 4 semaines successives ;

- une prairie de ray-grass anglais Reveille ayant reçu avant chaque cycle 40 ou $80 \mathrm{~kg} \mathrm{~N} / \mathrm{ha}$. Les ${ }^{\text {er }}$ et $2^{\mathrm{e}}$ cycles ont été étudiés durant 7 semaines et le $3^{\mathrm{e}}$ cycle durant 6 semaines consécutives;

- une prairie permanente normande ayant reçu avant les $2^{\mathrm{e}}$ et $3^{\mathrm{e}}$ cycles $4^{\circ}$ ou I $20 \mathrm{~kg} \mathrm{~N} / \mathrm{ha}$. Les $2^{\mathrm{e}}$ et $3^{\mathrm{e}}$ cycles ont été étudiés durant 4 semaines successives.

$2^{\circ}$ L'augmentation de la fertilisation azotée a peu modifié la composition morphologique des fourrages et les dates de réalisation des stades "début épiaison " et " début floraison " (fig. I, tabl. I). En revanche elle a, le plus souvent de façon significative, diminué la teneur en matière sèche des fourrages et augmenté leur teneur en matières azotées. Elle a eu tendance à augmenter les teneurs en cendres et en cellulose brute mais l'augmentation est rarement significative (fig. 2 et 3 , tabl. 2).

$3^{\circ}$ La fertilisation azotée a eu une action faible et de sens variable sur la digestibilité de la matière organique (fig. 2 et 3 , tabl. 2). Dans 25 comparaisons sur les 32 effectuées, la digestibilité n'a pas été modifiée, elle a été diminuée dans 3 cas et a été augmentée dans 4 cas.

En revanche, clle a augmenté, le plus souvent de façon significative, la digestibilité des matières azotées, mais la liaison entre la digestibilité et la teneur en matières azotées n'a pas été modifiée.

Ouant à la digestibilité de la cellulose brute, elle a été peu modifiée et a varié en général dans le même sens que la digestibilité de la matière organique.

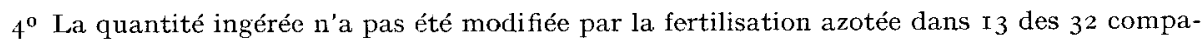
raisons; elle a été significativement augmentée de 6 p. Ioo en moyenne dans I I comparaisons et a été significativement diminuée de i I,7 p. Ioo en moyenne dans 8 comparaisons. Ces dernières ont toutes été effectuées sur des repousses de prairie permanente, aussi est-il difficile de savoir si la diminution de quantité ingérée résulte ou non d'une modification de la flore.

$5^{\circ}$ La fertilisation azotée a augmenté de façon importante la quantité de matière sèche, la 
quantité d'UF et surtout la quantité de matières azotées digestibles produite à l'hectare (tabl. 2, fig. 4 et 5 ).

$6^{\circ}$ Ces résultats, qui confirment ceux trouvés par d'autres auteurs, à savoir que la fertilisation azotée modifie peu la valeur énergétique des fourrages, sont discutés. En ce qui concerne la quantité ingérée, l'étude devra être reprise sur des prairies monospécifiques recevant des fumures azotées supérieures à Ioo $\mathrm{kg} \mathrm{N} / \mathrm{ha} / \mathrm{cycle}$.

\section{INTRODUCTION}

D'assez nombreuses études ont été publiées sur l'influence de la fertilisation azotée sur la valeur nutritive des fourrages. Tous les auteurs (BARTH et al., I959; Bratzler et al., I959 ; Chalupa et al., I961; Markley et al., I959; Poulton et al., I957; REID et al., I965 et I966) ont observé que la fertilisation azotée des graminées augmentait la teneur en matières azotées et par là, la digestibilité des matières azotées. En revanche, la fertilisation azotée a eu un effet variable sur la digestibilité de la matière sèche, de la matière organique ou de l'énergie. Elle n'a pas modifié la digestibilité des fourrages verts de ray-grass et de dactyle (Mrnson et al., I960) et de sorgho (REID et al., I964), des foins de première coupe de fléole (MAHONEY et Poulton, I962), de fétuque élevée et de dactyle (Reis et Jung, I965; REID et al., rg66) et des regains de dactyle (Poulton et al., I967); en revanche, elle a diminué la digestibilitéddes regains de fléole (Poulton et al., I963; WOELFEL et Poulton, I960) et de fléole + chiendent (CAMERoN 1966 et I967), et elle a augmenté la digestibilité des foins de dactyle (Washko et Mariotr, I960) (Markley et al., I960) et des regains de brome (BARTH et al., I959; MaRKLEy et al., 1959) de fétuque élevée et de dactyle (REID et Jung, I965; Reid et al., I966) et d'alpiste roseau (Chalupa et al., I959) ; toutefois ces modifications de la digestibilité ont été faibles (comprises entre $-5,2$ et $+5,7$ points pour la matière sèche) exception faite d'une augmentation de la digestibilité de la matière sèche de II,9 points observée par REID et JUNG (I965) entre un regain de fétuque élevée n'ayant pas reçu d'azote et celui ayant reçu $504 \mathrm{~kg}$ d'azote/ha sous forme d'urée avant la première coupe, (alors que la digestibilité du foin de première coupe avait été diminuée non significativement de 2,7 points).

En revanche, peu d'auteurs ont étudié l'action de la fertilisation azotée sur la quantité ingérée. La quantité ingérée par des bœufs (HoLmEs et LANG, I963; CAMEron, I966) et par des moutons (Mahoney et Poulton, ig62; Cameron, i967; REID et JUNG, I965; REID et al., I966) n'a pas été modifiée par la fertilisation azotée ; cependant quand REID et al. (déjà cités) offrirent, en libre choix, les foins aux moutons, ceux-ci délaissèrent presque entièrement les foins ayant reçu les fertilisations les plus élevées.

La majorité đe ces études ayant été faites sur des foins et des regains fauchés à un seul stade de végétation, on peut se demander si les différences observées entre les auteurs ne sont pas dues aux différences dans le stade de végétation choisi pour la coupe. Nous avons donc comparé en continu tout au long des cycles successifs de végétation l'influence de la fertilisation azotée sur la digestibilité de l'herbe verte et 1a quantité qui en est ingérée par le mouton, la fertilisation azotée adoptée étant proche de celle pratiquée par les agriculteurs, soit entre 40 et $120 \mathrm{~kg}$ d'azote $/$ ha par cycle. L'étude a porté au total sur 23 comparaisons. 


\section{MATÉRIEL ET MÉTHODES}

Trois prairies ont été étudiées :

En I966, au Centre de Recherches zootechniques et vétérinaires de Theix près de ClermontFerrand, une prairie de ray-grass Melle Pâture, ayant reçu avant le premier cycle de végétation $50 \mathrm{~kg}$ d'azote et $100 \mathrm{~kg}$ de $\mathrm{P}$ et $\mathrm{K}$ par hectare a été fauchée au stade épiaison. Elle a alors été divisée en 2 parcelles qui ont reçu $5^{\circ}$ et $100 \mathrm{~kg}$ d'azote par hectare. Les repousses du $2^{\mathrm{e}}$ cycle ont été étudiées du 8 août au 2 septembre soit entre des âges de 46 et 72 jours.

En 1967, au domaine du Pin-aux-Haras en Normandie, une prairie de ray-grass anglais Reveille ayant reçu à l'automne précédent $100 \mathrm{~kg}$ de $\mathrm{P}$ et $\mathrm{K}$ a été divisée en fin d'hiver en 2 parcelles qui ont été fertilisées avant le premier cycle et après les fauches préparatoires des $2^{\mathrm{e}}$ et $3^{\text {e }}$ cycles avec 40 ou $80 \mathrm{~kg}$ d'azote à l'hectare. Le premier cycle a été étudié du 7 mai au 23 juin soit du stade feuillu jusqu'à la pleine floraison; les repousses du deuxième cycle, correspondant à une fauche du premier cycle au stade pleine épiaison le 5 juin, ont été étudiées du 9 juillet au 25 août soit entre des âges de 34 et $7^{8}$ jours, et les repousses du $3^{\mathrm{e}}$ cycle, correspondant à une fauche du $2^{\mathrm{e}}$ cycle le 4 août ( 57 jours de reponsse), ont été étudićes du i I septembre au 20 octobre soit entre des âges de 38 et 77 jours.

En I968, une prairie naturelle du Pin-au-Haras ayant reçu en fin d'hiver $30 \mathrm{~kg}$ d'azote et $80 \mathrm{~kg}$ de $\mathrm{P}$ et $\mathrm{K}$ a été fauchée le 7 juin au stade pleine épiaison. Elle a alors été divisée en 2 parcelles qui ont reçu 40 ou $120 \mathrm{~kg}$ d'azote avant chacune des 2 exploitations suivantes. Les repousses du $2^{\mathrm{e}}$ cycle ont été étudiées du 29 juillet au 23 août soit entre des âges de 52 et 77 jours et les repousses du $3^{\text {e }}$ cycle du 12 octobre au 8 novembre soit entre des âges de 54 et 80 jours.

La digestibilité et la quantité ingérée de l'herbe de chaque traitement ont été mesurées sur des lots de 4 moutons mâles castrés de race Texel, âgés de 2 ans environ. A l'intérieur de chaque cycle, les mesures ont été continues, chaque période de mesure allant du lundi au samedi, étant séparée de la suivante par un seul jour, le dimanche. Avant chaque série de mesure, la période de préparation des animaux a été de 8 jours. Les moutons, maintenus en cages à métabolisme ont été alimentés à volonté ( 5 à Io $\mathrm{p}$. roo de refus) en 3 repas par jour à $7 \mathrm{~h} 30, \mathrm{I}_{3} \mathrm{~h} 30$ et $\mathrm{I} 7$ heures. L'herbe nécessaire aux animaux a été fauchée chaque matin sauf le dimanche. Pour limiter le tri effectué par les animaux, elle a été hachée en brins de 3 à $5 \mathrm{~cm}$ de longueur, quand elle contenait des tiges.

Les surfaces et les quantités fauchées ont été mesurées chaque jour. Au milieu de chaque période, un échantillon de $500 \mathrm{~g}$ environ a été prélevé ; les plantes étrangères (trèfle blanc et adventices) ont été enlevées, séchées et pesées ; les plantes étudiées restantes ont été séparées en limbes, tiges + gaines, épis et parties mortes (débris). Ces différentes fractions ont été séchées à l'étuve puis pesées et leur poids a été exprimé en p. Ioo du poids sec total des plantes.

Les échantillons représentatifs de l'herbe offerte, de l'herbe refusée et des fèces correspondant à chaque période de mesure ont été analysés pour déterminer leurs teneurs en cendres, en matières azotées et en cellulose brute Weende. La valeur énergétique de l'herbe a été estimée à partir des teneurs en matière organique digestible (MOD) et indigestible (MOND) par la formule de Breirem (1954):

$$
\mathrm{UF} / \mathrm{kg} \text { de matière sèche }=\frac{2,36 \mathrm{MOD}-\mathrm{r}, 20 \text { MOND }}{165^{\circ}}
$$

\section{RÉSULTATS}

\section{Composition morphologique et chimique}

L'augmentation de la fertilisation azotée n'a pas modifié les dates de réalisation des stades "début épiaison " (Ig mai pour 40 et $80 \mathrm{~kg}$ de $\mathrm{N} / \mathrm{ha}$ ) et " début floraison " (I4 juin pour $80 \mathrm{~kg}$, I6 juin pour $40 \mathrm{~kg}$ par ha) du ray-grass anglais Reveille au premier cycle de végétation. Elle a de même peu modifié la participation relative de différents organes à la matière sèche de la plante au cours des différents cycles de végé- 
tation de ce ray-grass (fig. I) ; la seule différence appréciable est l'augmentation de la part des parties mortes (débris) pour les premiers et deuxièmes cycles de végétation, consécutive vraisemblablement à l'augmentation de la production végétale (en moyenne respectivement I I Io $\mathrm{kg}$ et $946 \mathrm{~kg}$ de matière sèche à $1^{\prime}$ hectare au I ${ }^{\text {er }}$ et $2^{\mathbf{e}}$ cycles). La proportion de partie morte n'a pas été augmentée au $3^{e}$ cycle, soit parce que la production végétale n'a elle-même que faiblement augmenté ( $422 \mathrm{~kg}$ de matière sèche/ha en moyenne) soit parce que la plante la plus fertilisée a mieux résisté aux attaques de rouille, fréquentes à l'automne.
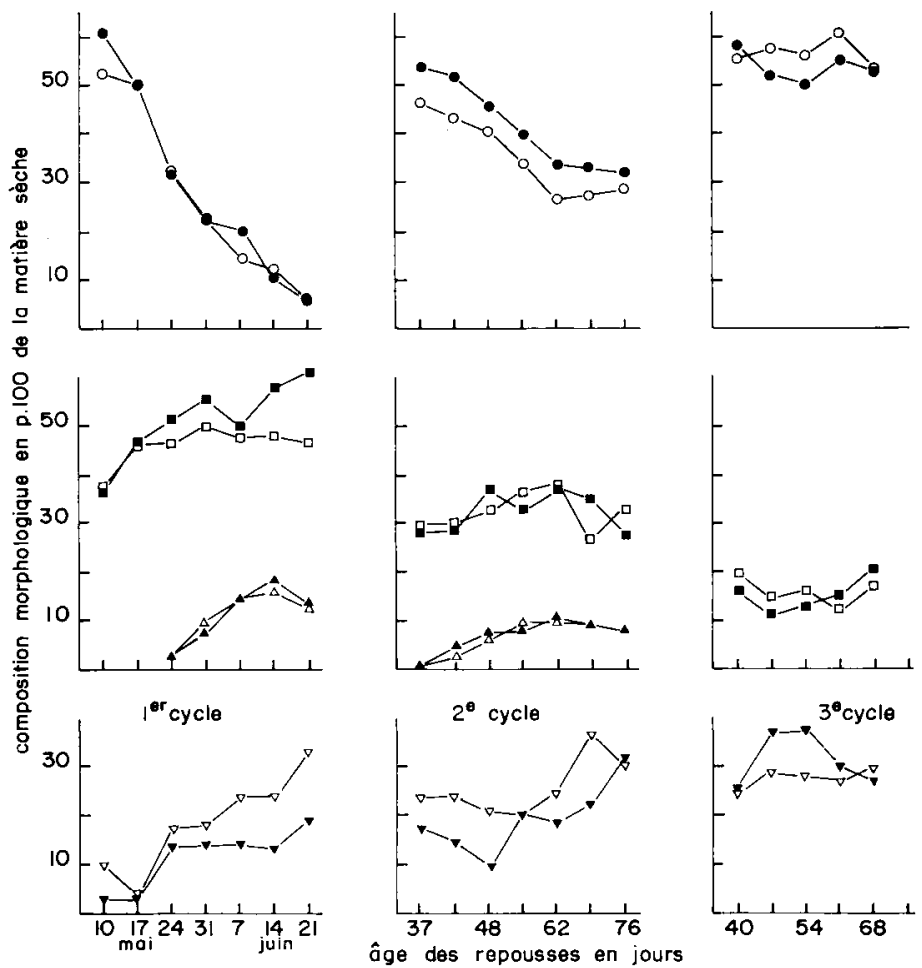

FIG. I. - Évolution avec l'âge au cours des premier, deuxième et troisième cycles de végétation, de la composition morphologique du Ray-grass anglais Reveille ayant regu 40 ou $80 \mathrm{~kg} N / \mathrm{cycle} / \mathrm{ha}$

$\begin{array}{lcc} & 40 \mathrm{~kg} \mathrm{~N} & 80 \mathrm{~kg} \mathrm{~N} \\ \text { Limbes } & \bullet & 0 \\ \text { Tiges } & - & \square \\ \text { Épis } & \mathbf{v} & \triangle \\ \text { Débris } & \nabla & \nabla\end{array}$

De même la fertilisation azotée a peu modifié la composition morphologique des repousses de $2^{\mathrm{e}}$ cycle du ray-grass $M$ elle et des repousses de $2^{\mathrm{e}}$ et $3^{\mathrm{e}}$ cycles de la prairie permanente (tabl. I) exception faite pour ces dernières d'une augmentation de la proportion de débris au $2^{\mathbf{e}}$ cycle au détriment des limbes verts et d'une diminution au $3^{\mathrm{e}}$ cycle, vraisemblablement pour les mêmes raisons que pour le ray-grass Reveille.

En revanche, la fertilisation azotée a diminué la proportion de trèfle blanc ou a limité son développement dans les prairies qui en contenaient peu au départ. C'est 
ainsi que la proportion de trèfle blanc dans la prairie de ray-grass Reveille a représenté en moyenne aux $\mathrm{I}^{\mathrm{er}}, 2^{\mathrm{e}}$ et $3^{\mathrm{e}}$ cycles de végétation respectivement, 0,$9 ; 2,8$ et 13,8 p. Ioo de la production de matière sèche de la parcelle ayant reçu $40 \mathrm{~kg} \mathrm{~N} / \mathrm{ha} /$ cycle contre 0,$6 ;$ I,7 et $2,2 \mathrm{p}$. Ioo de la production de la parcelle ayant reçu $80 \mathrm{~kg} \mathrm{~N} / \mathrm{ha} /$ cycle.

\section{TABLEAU I}

Composition morphologique moyenne des fourrages de $2^{\mathrm{e}}$ cycle de ray-grass Melle et des fourrages de $2^{\mathrm{e}}$ et $3^{\mathrm{e}}$ cycles de prairie permanente étudiés

\begin{tabular}{|c|c|c|c|c|c|c|c|}
\hline \multirow{2}{*}{$\begin{array}{l}\text { Nature du } \\
\text { fourrage }\end{array}$} & \multirow{2}{*}{$\begin{array}{c}\text { Numéro } \\
\text { du } \\
\text { cycle }\end{array}$} & \multirow{2}{*}{$\begin{array}{l}\text { Niveau de } \\
\text { fertilisation } \\
\mathrm{kg} \mathrm{N} / \mathrm{ha}\end{array}$} & \multirow{2}{*}{$\begin{array}{c}\text { Adventices } \\
(\%)\end{array}$} & \multirow{2}{*}{$\begin{array}{c}\text { Trèfle } \\
\text { blanc } \\
(\%)\end{array}$} & \multicolumn{3}{|c|}{$\begin{array}{c}\text { Composition morphologique } \\
\text { des graminées } \\
\text { (en } \% \text { de matière sèche) }\end{array}$} \\
\hline & & & & & $\begin{array}{c}\text { Limbes } \\
\text { verts }\end{array}$ & $\begin{array}{l}\text { Tiges }+ \\
\text { gaines } \\
\text { vertes }\end{array}$ & $\begin{array}{l}\text { Parties } \\
\text { mortes } \\
\text { (débris) }\end{array}$ \\
\hline \multirow{2}{*}{$\begin{array}{c}\text { Ray-grass } \\
\text { anglais Melle }\end{array}$} & \multirow[t]{2}{*}{2} & 50 & 5,0 & 0 & 72,0 & 14,6 & 13,4 \\
\hline & & 100 & 1,3 & 0 & 68,0 & 18,4 & 13,6 \\
\hline \multirow{4}{*}{$\begin{array}{c}\text { Prairie } \\
\text { naturelle }\end{array}$} & \multirow{2}{*}{2} & 40 & 2,9 & 7,3 & 38,4 & 23,6 & 38,0 \\
\hline & & 120 & 0 & 1,6 & 26,5 & 26,0 & 47,5 \\
\hline & \multirow{2}{*}{3} & 40 & 0 & 3,7 & 32,4 & 11,7 & 55,9 \\
\hline & & 120 & 0 & 0 & 35,5 & 15,2 & 49,3 \\
\hline
\end{tabular}

L'influence de la fertilisation azotée sur la composition chimique des fourrages est présentée dans les figures 2 et 3 et dans le tableau 2 pour tous les fourrages étudiés. La fertilisation azotée a, le plus souvent de façon significative, diminué la teneur en matière sèche des fourrages et augmenté leur teneur en matières azotées excepté pour le $3^{\text {e }}$ cycle de ray-grass Reveille, ce qui s'explique par le développement du trèfle blanc dans la parcelle n'ayant reçu que $40 \mathrm{~kg} \mathrm{~N} / \mathrm{ha} /$ cycle. Les teneurs en cendres et en cellulose tendent elles aussi à augmenter avec la fertilisation azotée mais l'augmentation est rarement significative (tab1. 2). En ce qui concerne la teneur en cendres, le $3^{\text {e }}$ cycle de ray-grass Reveille fait encore exception, ainsi que le $3^{\mathrm{e}}$ cycle de prairie permanente. Pour ce dernier la raison doit en être une contamination importante du fourrage par la terre lors des opérations de fauche et de ramassage, contamination d'ailleurs montrée par les teneurs en cendres très élevées, et qui a dû être d'autant plus importante que la production végétale était faible.

Nous avons déterminé les teneurs en glucides solubles des échantillons de raygrass Melle. Ceux ayant reçu Ioo $\mathrm{kg} \mathrm{N} /$ ha ont été moins riches en glucides solubles que ceux ayant reçu $50 \mathrm{~kg} \mathrm{~N} /$ ha ; en moyenne I I,7 contre I3,0 p. Ioo. La différence, I,3 point, n'est pas significative, mais il est vrai que la différence de teneurs en matières azotées, $+\mathrm{I}, 7$ point, ne l'est pas non plus. 


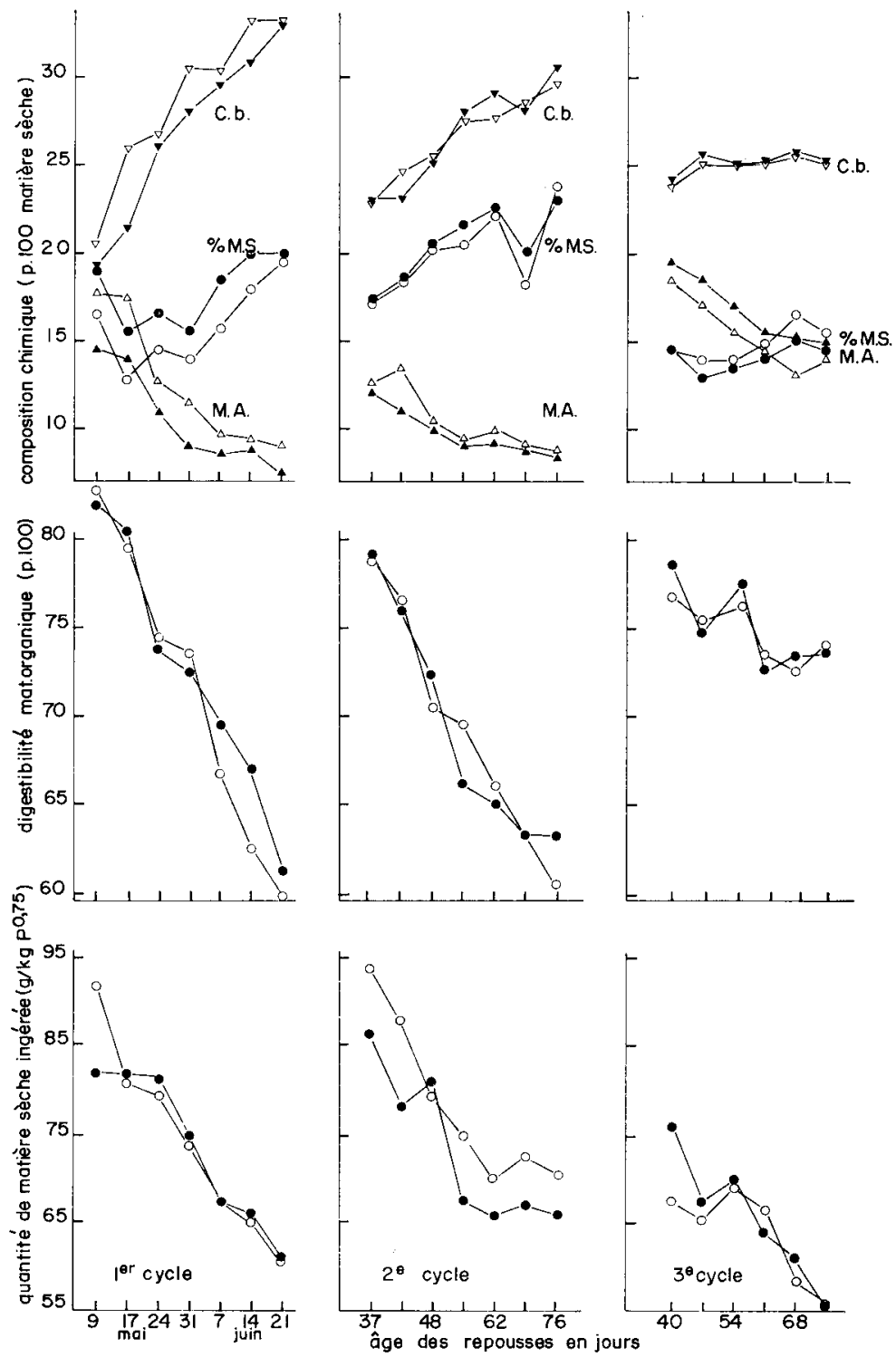

FIg. 2. - Evvolution avec l'âge au cours des premier, deuxième et troisième cycles de végétation, de la composition chimique, de la digestibilité de la matière organique et de la quantité de matière sèche ingérée du Ray-grass anglais Reveille ayant vęu 40 ou 80 N/cycle/ha.

$\begin{array}{llll}40 \mathrm{~kg} \mathrm{~N} / \text { cycle } / \mathrm{ha}: & \bullet & \Delta & \boldsymbol{\nabla} \\ 80 \mathrm{~kg} / \mathrm{N} / \text { cycle } / \mathrm{ha}: & 0 & \Delta & \nabla\end{array}$ 

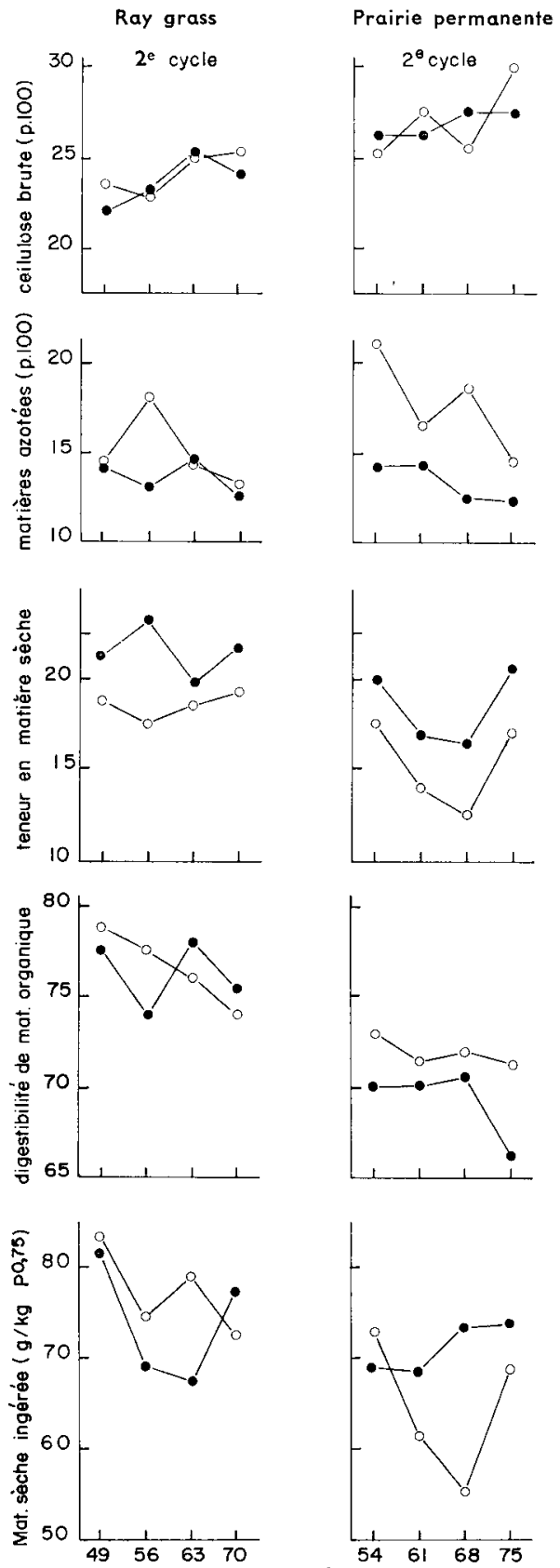

\section{Prairie permanente}
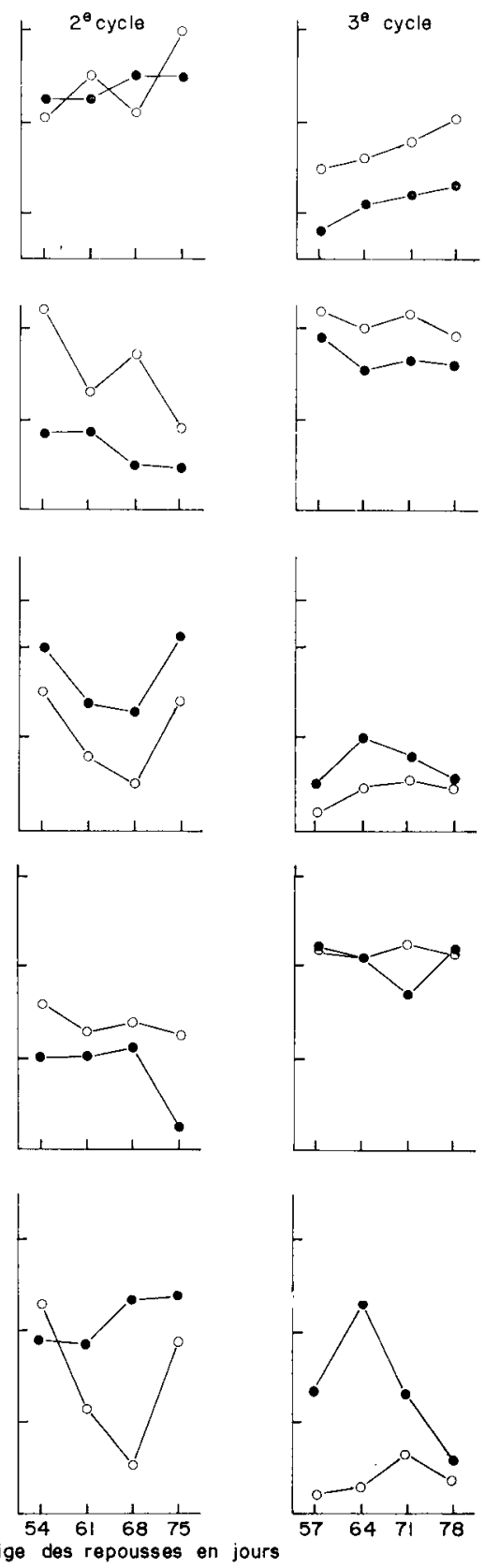

FIG." 3. - Évolution avec l'âge de la composition chimique, de la digestibilité et de la quantité ingérée d'un deuxième cycle de Ray-grass Melle ayant reç $50(\bullet)$ ou $100(0) \mathrm{kg} N /$ ha et de l'herbe d'une prairie permanente ayant recu $40(\bullet)$ ou 120 (०) $\mathrm{kg} \mathrm{N}$ /cycle/ha. 
Influence de la fertilisation azotée sur lo la quantité ingérée et la production à l'hectar

\begin{tabular}{|c|c|c|c|c|}
\hline & \multicolumn{4}{|c|}{ Ray-grass anglais Reveille } \\
\hline & $\mathrm{kgN} / \mathrm{ha}$ & 1er cycle & $2^{\mathrm{e}}$ cycle & $3^{\mathrm{e}}$ cycle \\
\hline \multicolumn{5}{|l|}{ Teneur en $(\%)$} \\
\hline Natière sèche & $\begin{array}{l}40 \\
80\end{array}$ & $17,9 \neq-2,1 * *$ & $\begin{array}{l}20,5 \\
20,0\end{array} \neq \rightarrow 0,5$ & $\begin{array}{l}14,0 \\
14,8\end{array} \neq+0,8 * *$ \\
\hline Cendres & $\begin{array}{l}40 \\
80\end{array}$ & $\frac{7, l_{4}}{8,4} \neq+1,0^{* *}$ & $\underset{9,4}{9,1} \neq \rightarrow \quad 0,3$ & $\begin{array}{l}11,8 \\
10,8\end{array} \neq-1,0$ \\
\hline Matières azotées & $\begin{array}{l}40 \\
80\end{array}$ & $\frac{10,4}{12,5} \neq+2,1^{* *}$ & $\frac{9,1}{10,5} \neq+0,6$ & ${ }_{15,3}^{16,4} \neq-1,1$ \\
\hline Cellulose brute & $\begin{array}{l}40 \\
80\end{array}$ & $\frac{26,8}{28,5} \neq+1,7$ & $\begin{array}{l}26,7 \\
26,9\end{array} \neq+0,2$ & $\begin{array}{l}24,4 \\
24,9\end{array} \neq+0,5$ \\
\hline \multicolumn{5}{|l|}{$\begin{array}{l}\text { Coefficient de } \\
\text { digestibilité }(\%)\end{array}$} \\
\hline Matière organique & $\begin{array}{l}40 \\
80\end{array}$ & $\begin{array}{l}72,3 \\
71,4\end{array} \neq-0,9(1)$ & $\begin{array}{l}69,4 \\
69,2\end{array}-0,2$ & $\begin{array}{l}75,1 \\
74,7\end{array}-0,4$ \\
\hline Matières azotées & $\begin{array}{l}40 \\
80\end{array}$ & $\frac{53,1}{61,7} \neq+8,6^{* *}$ & $\begin{array}{l}54,2 \\
58,2\end{array}$ & $\begin{array}{l}68,2 \\
67,2\end{array}-1,0$ \\
\hline Cellulose brute & $\begin{array}{l}40 \\
80\end{array}$ & $70,9 \neq+1,8 *$ & $\begin{array}{l}63,1 \\
61,7\end{array} \longrightarrow 1,6$ & $\frac{73,3}{72,6} \neq-0,7$ \\
\hline $\begin{array}{l}\text { Valeur énergétique } \\
\text { UF/kg M.S. }\end{array}$ & $\begin{array}{l}40 \\
80\end{array}$ & $\begin{array}{l}0,77 \\
0,7 / 1\end{array} \neq-0,03$ & $\begin{array}{l}0,70 \\
0,69\end{array} \neq-0,01$ & $\begin{array}{l}0,79 \\
0,79\end{array} \neq 0,00$ \\
\hline $\begin{array}{l}\text { Teneur en MAD } \\
\text { (g/kg M.S.) }\end{array}$ & $\begin{array}{l}10 \\
80\end{array}$ & ${ }_{80}^{58} \neq+22 * *$ & ${ }_{63}^{54} \neq+9$ & ${ }_{105}^{113} \neq-8$ \\
\hline $\begin{array}{l}\text { Quantité de M.S. } \\
\text { ingérée }(\mathrm{g} / \mathrm{kg} P \quad 0,75)\end{array}$ & $\begin{array}{l}40 \\
80\end{array}$ & ${ }_{74,3}^{73,6} \neq+0,7$ & $\frac{73,2}{78,4} \neq+5,2 * *$ & $65,6 \neq-1,9$ \\
\hline \multicolumn{5}{|l|}{ Production à l'hectare } \\
\hline Matière sèche (t.) & $\begin{array}{l}40 \\
80\end{array}$ & $\frac{5,21}{6,32} \neq+1,11 * * *$ & $\begin{array}{l}2,45 \\
3,40\end{array} \neq+0,95 * *$ & $\begin{array}{l}1,66 \\
1,81\end{array} \neq+0,15$ \\
\hline $\mathrm{UF}$ & $\begin{array}{l}40 \\
80\end{array}$ & $\begin{array}{l}3700 \\
4360\end{array} \neq+660^{*}$ & $\begin{array}{l}1670 \\
2280\end{array} \neq+610^{* *}$ & $\begin{array}{l}1320 \\
1420\end{array} \neq+100$ \\
\hline MAD $(\mathrm{kg})$ & $\begin{array}{l}10 \\
80\end{array}$ & ${ }_{435}^{246} \neq+189 * * *$ & $\frac{128}{202} \neq+7 z * *$ & $\begin{array}{l}187 \\
187\end{array}$ \\
\hline
\end{tabular}

(1) La diminution de la digestibilité de la matière organique a été significative $(\mathrm{P}<0,05)$ pour les trois

(2) L'augmentation de quantité ingérée a été significative $(\mathrm{P}<0,05)$ pour les 3 premières des 4 semaines de

(3) La diminution de quantité ingérée a été significative $(\mathrm{P}<0,05)$ pour les 3 dernières des 4 semaines de

* significatif à 5 p. 100

** significatif à 1 p. 100

*** significatif à 1 p. 1000 
composition chimique, la valeur nutritive,

valeurs moyennes par cycle de végétation)

Ray-grass anglais Melle Pâture

$\mathrm{kgN} / \mathrm{ha}$ $2^{\mathrm{e}}$ cvcle
Prairie permanente

2 e cycle $3^{\mathrm{e}}$ cycle

\begin{tabular}{|c|c|c|c|c|}
\hline $\begin{array}{r}50 \\
100\end{array}$ & $\frac{21,5}{18,5} \neq-3,0 *$ & $\begin{array}{r}40 \\
120\end{array}$ & $\begin{array}{l}18,5 \\
15,2\end{array} \neq-3,3 *$ & $\begin{array}{l}13,6 \\
12,0\end{array}-1,6^{*}$ \\
\hline $\begin{array}{r}50 \\
100\end{array}$ & $\begin{array}{l}10,8 \\
11,3\end{array} \neq+0,5$ & $\begin{array}{r}40 \\
120\end{array}$ & ${ }_{9,2}^{8,4} \neq+0,8$ & $\begin{array}{c}19,3 \\
15,4\end{array} \neq-3,9$ \\
\hline $\begin{array}{r}50 \\
100\end{array}$ & $\begin{array}{l}13,3 \\
15,0\end{array} \neq+1,7$ & $\begin{array}{r}40 \\
120\end{array}$ & $\frac{13,2}{17,5} \neq+4,3 *$ & ${ }_{20,3}^{18,3} \neq+2,0^{* *}$ \\
\hline $\begin{array}{r}50 \\
100\end{array}$ & $\begin{array}{l}23,7 \\
24,1\end{array} \neq+0,4$ & $\begin{array}{r}40 \\
120\end{array}$ & $\begin{array}{l}26,8 \\
27,0\end{array} \neq+0,2$ & ${ }_{23,7}^{20,5} \neq+3,2 * * *$ \\
\hline
\end{tabular}

\begin{tabular}{|c|c|c|c|c|}
\hline $\begin{array}{r}50 \\
100\end{array}$ & $\frac{76,2}{76,6} \neq+0,4$ & $\begin{array}{r}40 \\
120\end{array}$ & $\begin{array}{l}69,2 \\
72,0\end{array}$ & $\begin{array}{l}75,3 \\
75,9\end{array}$ \\
\hline $\begin{array}{r}50 \\
100\end{array}$ & $\begin{array}{l}65,6 \\
68,1\end{array}$ & $\begin{array}{r}40 \\
120\end{array}$ & $\begin{array}{l}64,4 \\
73,1\end{array} \neq+8,7 *$ & $\begin{array}{l}73,9 \\
75,1\end{array} \neq+1,2$ \\
\hline $\begin{array}{r}50 \\
100\end{array}$ & $\begin{array}{l}71,4 \\
74,8\end{array}$ & $\begin{array}{r}40 \\
120\end{array}$ & ${ }_{69,4}^{66,1} \neq+3,3$ & $\frac{72,3}{77,8} \neq+3,5$ \\
\hline $\begin{array}{r}50 \\
100\end{array}$ & $\begin{array}{l}0,82 \\
0,82\end{array} \neq \quad 0,00$ & $\begin{array}{r}40 \\
120\end{array}$ & $\begin{array}{l}0,70 \\
0,75\end{array}$ & $\begin{array}{l}0,72 \\
0,77\end{array} \neq+0,05$ \\
\hline $\begin{array}{r}50 \\
100\end{array}$ & $\underset{104}{88} \neq+16$ & $\begin{array}{r}40 \\
120\end{array}$ & $\begin{array}{r}86 \\
129\end{array} \neq+43 *$ & $137 \neq+16 * *$ \\
\hline $\begin{array}{r}50 \\
100\end{array}$ & $74,0 \neq+3,5\left(^{2}\right)$ & $\begin{array}{r}40 \\
120\end{array}$ & $\begin{array}{l}71,2 \\
64,8\end{array} \neq-6,4\left({ }^{3}\right)$ & $\begin{array}{l}63,6 \\
53,9\end{array}$ \\
\hline $\begin{array}{r}50 \\
100\end{array}$ & $\begin{array}{l}2,74 \\
3,94\end{array} \neq+1,20^{*}$ & $\begin{array}{r}40 \\
120\end{array}$ & ${ }_{3,86}^{2,34} \neq+1,52 * *$ & ${ }_{2,45}^{1,55} \neq+0,88 * *$ \\
\hline $\begin{array}{r}50 \\
100\end{array}$ & $\begin{array}{l}2370 \neq+830 * \\
3200 \neq+8\end{array}$ & $\begin{array}{r}40 \\
120\end{array}$ & $\begin{array}{l}1620 \\
2850\end{array} \neq+1230 * *$ & $\begin{array}{l}1120 \\
1870\end{array}$ \\
\hline $\begin{array}{r}50 \\
100\end{array}$ & $\frac{241}{407} \neq+166 * *$ & $\begin{array}{r}40 \\
120\end{array}$ & $498 \neq+290 * *$ & $\frac{212}{372} \neq+160 * *$ \\
\hline
\end{tabular}

dernières périodes (semaines) du premier cycle. mesure. mesure. 


\section{Digestibilité, valeur nutritive et quantité ingérée}

La fertilisation azotée a eu une action faible et de sens variable sur la digestibilité de la matière organique (fig. 2 et 3 , tabl. 2). Elle n'a pas modifié la digestibilité du premier cycle de ray-grass Reveille exploité entre le stade feuillu et le stade pleine épiaison (4 premières semaines) mais l'a diminué significativement $(P<0,05)$ de 2,8 points en moyenne pour les stades plus tardifs, pleine épiaison, pleine floraison, soit durant les 3 dernières semaines. Elle n'a pas modifié significativement la digestibilité des repousses de $2^{\text {e }}$ cycle des 2 ray-grass Reveille et Melle Pâture et de $3^{\text {e }}$ cycle de la prairie permanente et $\mathrm{d} u$ ray-grass Reveille; elle a significativement $(\mathrm{P}<0,05)$ augmenté, de 2,8 points en moyenne, la digestibilité des repousses de $2^{\text {e }}$ cycle de la prairie permanente, sans qu'il soit possible de dire s'il s'agit d'une action directe ou par l'intermédiaire d'une modification de la flore. En définitive la digestibilité n'a pas été modifiée dans 25 comparaisons sur les 32 effectuées ; elle a été diminuée 3 fois et elle a été augmentée 4 fois.

Comme on pouvait s'y attendre la fertilisation azotée a augmenté le plus souvent de façon significative, exception faite du $3^{\mathrm{e}}$ cycle de ray-grass, la digestibilité des matières azotées puisqu'on sait que cette dernière varie dans le même sens que la teneur en matières azotées. La fertilisation azotée n'a d'ailleurs pas modifié les liaisons entre la digestibilité des matières azotées et la teneur en matières azotées puisque la teneur en matières azotées indigestibles des fourrages ayant reçu la plus faible fertilisation azotée a été très voisine de celle des fourrages ayant reçu la fertilisation azotée la plus forte ; respectivement $4,82 \pm 0,08$ contre $4,74 \pm 0,09$ pour $n=32$. La digestibilité de la cellulose brute a été peu modifiée et a varié en général dans le même sens que la digestibilité de la matière organique.

I a valeur énergétique nette des fourrages, exprimée en UF par $\mathrm{kg}$ de matière sèche, a été peu modifiée par la fertilisation azotée. Ėlle n'a augmenté de façon significative, et de 0,05 UF seulement, que pour le deuxième cycle de la prairie permanente. Fn revanche, la teneur en matières azotées digestibles a augmenté (en moyenne de 9 à $43 \mathrm{~g}$ suivant les cycles) avec la fertilisation azotée (sauf celle du $3^{\mathrm{e}}$ cycle de raygrass) le plus souvent de façon significative (tabl. 2).

La quantité ingérée n’a pas été modifiée par la fertilisation lors des I er et $3^{\text {e }}$ cycles de ray-grass Reveille, elle a été significativement augmentée de 6 p. roo en moyenne lors du $2^{\mathrm{e}}$ cycle des deux ray-grass Reveille et Melle Pâture, mais elle a été significativement diminuée de $\mathrm{I}, 7 \mathrm{p}$. Ioo en moyenne lors des $2^{\mathrm{e}}$ et $3^{\mathrm{e}}$ cycles de la prairie permanente (tab1. 2 , fig. 2 et 3 ). Dans le cas de la prairie permanente il est cependant difficile de savoir si la diminution résulte ou non d'une modification de la flore.

\section{Production à l'hectare}

La quantité de matière sèche produite par hectare a été augmentée de façon importante et significative par la fertilisation azotée, sauf pour le $3^{\text {e }}$ cycle de ray-grass Reveille où le supplément de production n'a été que de I50 kg/ha (tabl. 2 , fig. 4 et 5). Le développement du Trèfle blanc dans la parcelle la moins fertilisée a donc eu sur la production une action pratiquement équivalente à un supplément de fumure de $40 \mathrm{~kg} \mathrm{~N} / \mathrm{ha}$. De même, la fertilisation azotée a permis d'augmenter de façon importante les quantités d'unités fourragères (UF) et surtout de matières azotées diges- 


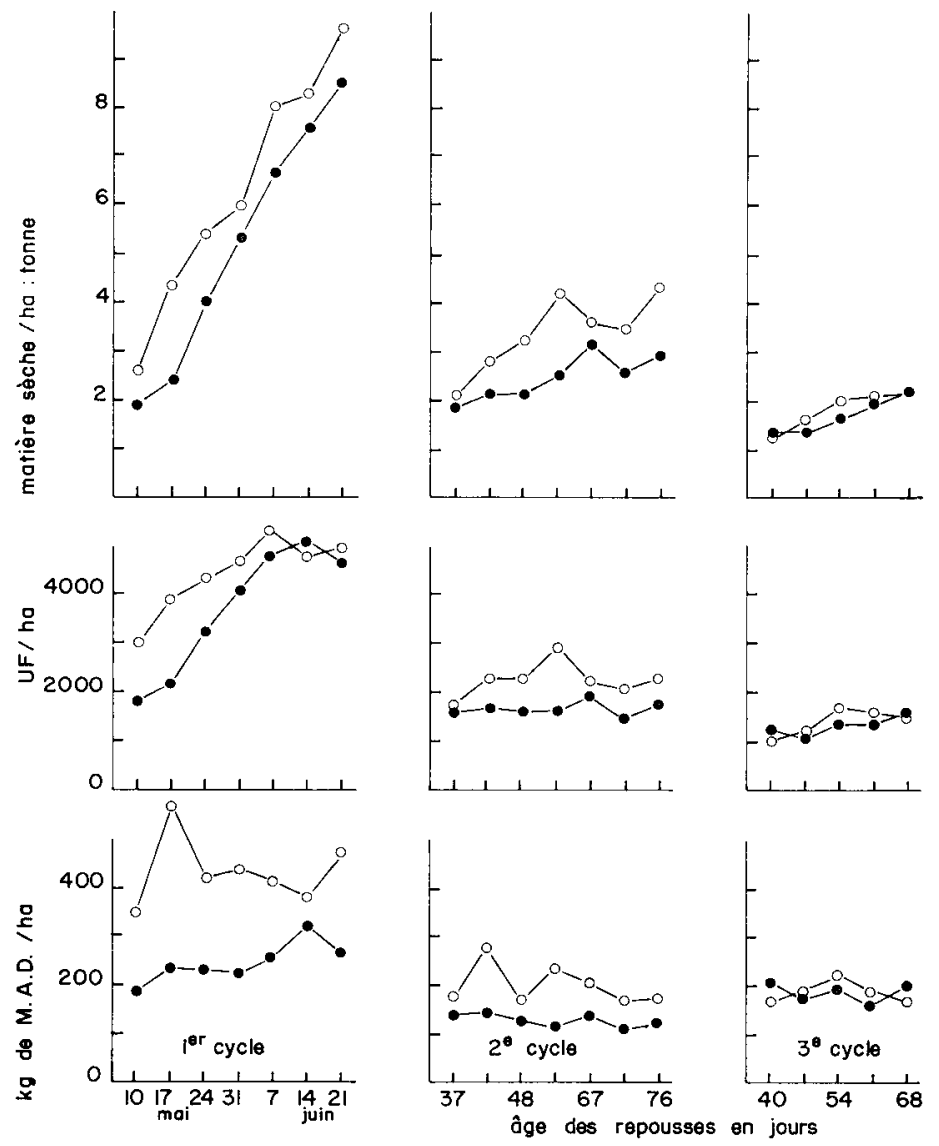

FIG. 4. - Évolution avec l'âge au cours de trois cycles de végétation de la quantité de matière sèche, d'UF et de matières azotées digestibles produites à l'hectare par le Ray-grass anglais Reveille ayant reçu $40(\bullet)$ ou $80 \mathrm{~kg}(\mathrm{O})$ d'azote à l'hectare avant chaque cycle.

tibles (MAD) produites à 1'hectare. Par kg d'azote supplémentaire, l'augmentation a été de $28 \mathrm{~kg}$ de matière sèche, I6,5 UF et 4,7 kg de MAD pour le I er cycle de raygras, de 24,0, 24, 0 et I $9,0 \mathrm{~kg}$ de matière sèche, I5,2, I6,6 et I5,4 UF et de I,8, 3,3 et $3,6 \mathrm{~kg}$ de MAD respectivement pour les $2^{\circ}$ cycles de ray-grass Reveille, de ray-grass Melle et de prairie permanente, et de 3,7 et $\mathrm{I} I, 0 \mathrm{~kg}$ de matière sèche, 2,5 et 9,4 UF et o et $2 \mathrm{~kg}$ de MAD pour les $3^{\text {e }}$ cycles de ray-grass Reveille et de prairie permanente. Il apparaît donc que malgré une fertilisation azotée plus importante la réponse de la prairie permanente à la fumure a été aussi importante que celle du ray-grass anglais.

\section{DISCUSSION}

N'avançant pas ou peu les dates de réalisation des stades de végétation tardifs (début épiaison, début floraison) comme l'avait déjà montré Minson et al. (I960), il est normal que la fertilisation azotée n'ait modifié que très peu la composition 

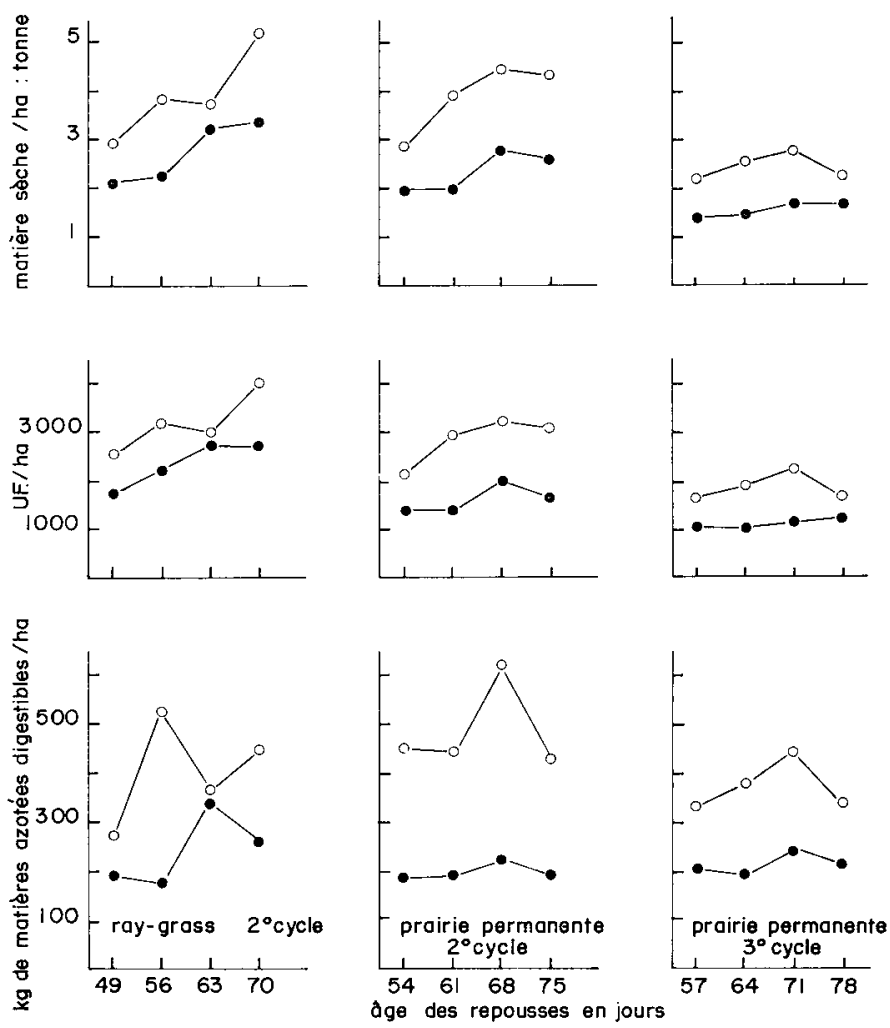

FIG. 5. - Évoiution avec l'âge de la quantité de matière sèche, d'UF et de matières azotées digestibles produites $\dot{a}$ l'hectare par un deuxième cycle de Ray-grass Melle Pâture ayant reçu $50(\bullet)$ ou $10 G$ (o) kg N/ha et par un deuxième et un troisième cycle de prairie permanente ayant reçu 40 (•) ou 120 (०) $\mathrm{kg} N$ lha avant chaque cycle.

morphologique des plantes puisque cette dernière dépend avant tout du stade de végétation.

En revanche, la fertilisation azotée, en augmentant la teneur en matières azotées des fourrages, a augmenté aussi la digestibilité de ces matières azotées, ce qui avait déjà été trouvé par de nombreux auteurs (voir introduction). On sait que l'augmentation de la teneur en matières azotées par la fumure s'accompagne d'une diminution de la teneur en glucides solubles (WAITE I958, JONES et al., I96I) ; cela résulte du fait que l'élaboration des glucides solubles, limitée par la surface foliaire et la photosynthèse, n'est pas accrue par la fertilisation, alors que leur utilisation, pour la synthèse du supplément de matière sèche/ha entraînée par la fertilisation, 1'est. D'après nos résultats, l'augmentation de la teneur en matières azotées serait aussi associée à une légère augmentation de la teneur en cellulose brute, tout au moins pour les premiers cycles de croissance; la majorité des auteurs (cf. revue de BLASER, I964) ne l'ont cependant pas observée mais ils ont étudié surtout des regains; certains auteurs (MARKIEY et al., I959) ont même observé le phénomène inverse mais pour des fumures azotées bien supérieures à celles que nous avons employées : $252 \mathrm{~kg} \mathrm{~N} / \mathrm{ha}$ avant la coupe. 
Il n'est donc pas étonnant que la fumure azotée modifie peu la digestibilité de la matière organique et par là, la valeur énergétique des fourrages malgré l'augmentation de la teneur en matières azotées qu'elle entraîne, puisque celle-ci est compensée par une diminution de la teneur en glucides solubles, éléments entièrement digestibles, et est accompagnée pour les premiers cycles par une augmentation de la teneur en cellulose brute. Cette augmentation, quoique non significative, expliquerait peutêtre l'action dépressive de la fertilisation azotée sur la digestibilité des fourrages à la fin du premier cycle puisque la digestibilité de la cellulose brute, si elle est très élevée pour des fourrages jeunes, diminue rapidement avec le stade de végétation aul cours du premier cycle de croissance. Quoi qu'il en soit si la fertilisation peut avoir parfois et à certains stades, une action sur la digestibilité de la matière organique, celle-ci reste très limitée, ce qui est en accord avec les données de la plupart des auteurs.

Il est malheureusement plus difficile de conclure quant à l'action de la fertilisation azotée sur la quantité de fourrage ingérée. Si des fumures azotées inférieures à Ioo $\mathrm{kg} \mathrm{N} /$ ha/cycle ne semblent pas modifier la quantité ingérée et pourraient même parfois avoir tendance à l'augmenter légèrement, nous ne savons pas si la diminution de quantité ingérée observée pour l'herbe de prairie permanente ayant reçu I $20 \mathrm{~kg} \mathrm{~N} /$ cycle, résulte d'une diminution générale des qualités " gustatives " de la flore par suite d'une fumure trop élevée, ou d'une modification de la flore. Les résultats obtenus à l'étranger étant peu nombreux, l'étude devra être reprise sur des prairies monospécifiques.

Nos résultats montrent que pour une fertilisation inférieure ou égale à IoO-I $20 \mathrm{~kg} \mathrm{~N} / \mathrm{ha} /$ cycle, l'augmentation de la production de matière sèche/ha mesurée dans tous les essais classiques de fumure par les agronomes ou les vulgarisateurs traduit une augmentation du même ordre de la quantité d'unités fourragères produite à l'hectare. C'est ainsi que pour le ray-grass Reveille, en fauchant le premier cycle le 5 juin, le $2^{\mathrm{e}}$ le 4 août (comme nous l'avons fait pour préparer les repousses des $2^{\mathrm{C}}$ et $3^{\mathrm{e}}$ cycles) et le $3^{\mathrm{e}}$ cycle le $\mathrm{I}^{\mathrm{er}}$ octobre, nous avons obtenu avec celui ayant reçu $80 \mathrm{~kg} \mathrm{~N} /$ cycle, 3,2 tonnes de matière sèche soit 28,5 p. Ioo, I $985 \mathrm{UF}$ soit $25,4 \mathrm{p}$. IOO et $313 \mathrm{~kg}$ de matières azotées digestibles soit 59,5 p. Ioo de plus qu'avec celui ayant reçu $4^{\circ} \mathrm{kg} \mathrm{N} /$ cycle. La valeur alimentaire du fourrage récolté a, en outre, été indépendante de la fumure reçue : en moyenne respectivement pour les fumures 80 et $40 \mathrm{~kg} \mathrm{~N} /$ cycle $68,5 \mathrm{p}$. Ioo contre 69 , I p. Ioo pour le coefficient de digestibilité de la matière organique et 69,5 contre $66,7 \mathrm{~g} / \mathrm{kg} \mathrm{P}^{0,75}$ pour la quantité ingérée. Jusqu'à I $00 \mathrm{~kg} \mathrm{~N} /$ cycle, la fertilisation des graminées s'avère donc très bénéfique. Pourtant la fumure azotée reçue par les prairies françaises n'augmente que très lentement, bien plus lentement que celles reçues par les céréales et les plantes sarclées. C'est qu'en réalité le problème est complexe. S'il est facile d'accroître la production fourragère, il est beaucoup plus difficile de récolter correctement le supplément de production obtenue, l'augmentation de la production fourragère à l'unité de surface s'accompagnant presque toujours d'un accroissement des difficultés de récolte et de conservation et par là, d'une augmentation des pertes, tout au moins avec les techniques traditionnelles de récolte. Ceci explique, au moins en partie, l'intérêt suscité dans toutes les zones d'élevage, par la déshydratation. Il n'en est pas de même pour les prairies uniquement pâturées pour lesquelles la fertilisation azotée augmente peu ou pas les pertes à condition que les dates d'exploitation par le pâturage soient déterminées par la hauteur de l'herbe ou la quantité d'herbe présente à l'hectare. Dans ce 
cas la fertilisation azotée entraîne un supplément de production de matière sèche plus faible mais elle permet d'exploiter une herbe plus jeune et par là, plus digestible et ingérée en plus grande quantité. Ellle est alors entièrement justifiée.

Reçu pour publication en octobve $19 \% 0$.

\author{
SUMMARY \\ FEEDING VALUE OF GREEN FORAGES AS INFI,UENCED \\ BY NITROGEN FERTIIIZATION
}

I. A sustained comparison was made during all the successive growth cycles on the effects of nitrogen fertilization upon herbage intake and digestibility in sheep.

The study was carried out on :

- a meadow of perennial rye-grass Melle Pature, treated with 40 or $100 \mathrm{~kg} \mathrm{~N} / \mathrm{ha}$ after the ist cycle. The and cycle was studied during 4 successive weeks;

- a meadow of perennial rye-grass Reveille, treated with 40 or $80 \mathrm{~kg} \mathrm{~N} / \mathrm{ha}$ before each cycle. The Ist and 2nd cycles were studied during 7 weeks and the $3^{\text {rd }}$ cycle during 6 consecutive weeks ;

- a permanent Normand meadow treated with 40 or $120 \mathrm{~kg} \mathrm{~N} / \mathrm{ha}$ before the 2 nd and 3 rd cycles. These two cycles were studied during 4 consecutive weeks.

2. The increase of nitrogen fertilization did not modify very much neither the morphological composition of the forages nor the dates of appearance of the stages : early ear emergence and early flowering (fig. I, table I). But on the other hand, nitrogen fertilization often resulted in significant decrease in the dry matter content of the forage and in significant increase of the crude protein content. It also had a tendency to increase the ash and crude fiber content, but this was seldom significant (fig. 2 and 3 , table 3 ).

3. The effect of nitrogen fertilization upon digestibility of the organic matter was small and variable (fig. 2 and 3 , table 2). 25 comparisons out of 32 did not show any change of the digestibility ; in 3 cases there was a decrease and in 4 cases an increase.

On the other hand, nitrogen fertilization often resulted in significant increase of the digestibility of crude protein, but the relationship between digestibility and crude protein content was not modified.

The digestibility of crude fiber was only a little modified and generally varied in the same direction as the digestibility of the organic matter.

4. Nitrogen fertilization did not change the herbage intake in 13 comparisons out of 32 ; there was a significant mean increase of $6 \mathrm{p}$. Ioo in I I cases and a significant mean decrease of I I.7 p. 100 in 8 cases. For the latter, the comparisons were carried out on regrowths of permanent meadow, and it is therefore difficult to know if the decrease in the forage intake is or is not due to a change of the botanical composition of the meadow.

5. Nitrogen fertilization provoked an important increase in the amount of dry matter, FU, and above all digestible crude protein per hectare (table 2, fig. 4 and 5).

6. The results obtained, which confirm those of other authors according to whom nitrogen fertilization modifies only a little the energy value of the forages, are discusssd. As far as the forage intake is concerned, the study should be extended to monospecific meadows treated with amounts of nitrogen higher than too $\mathrm{kg} \mathrm{N} / \mathrm{ha} / \mathrm{cycle}$.

\title{
RÉFÉRENCES BIBLIOGRAPHIQUES
}

Barth K. M., Vander Noot G. W., Cason J. L., I959. A comparison of the nutritive value of alfalfa hay with bromegrass and reed canary grass hays at various levels of nitrogen fertilization. J. Nutr., 68, $383-69$ I.

Blaser R. E., r964. Symposium on forage utilization : effects of fertility levels and stage of maturity on forage nutritive value. J. Anim. Sci., 23, 246-253. 
Bratzler J. W., Keck E., Mariott Jr, L. li., Washko J. B., I959. Nutritive value of orchard grass as affected by level of nitrogen fertilization and stage of maturity (abstr). J. Dairy Sci., 42, 934.

Chalupa W. V., Cason J. L., Baumgardt B. R., I959. The nutritive value of reed canary grass hays grown under different levels of nitrogen fertilization (Abst). J. Dairy Sci., 42, 935.

Holmes J.-C., LANG R. W., I963. Effects of fertilizer nitrogen and herbage dry matter content on herbage intake and digestibility in bullocks. Anim. Prod., 5, I7-26.

Jones D. I. H., Griffith G., Walters R. J, K., I961. The effect of nitrogen fertilizer on the water soluble carbohydrate content of perennial rye grass and cocksfoot. J. Brit. Grassl. Soc., 16, 272.

Mahoney A. W., Poulton B. R., I962. Effects of nitrogen fertilization and date of harvest on the acceptability of timothy forage (Abstr). J. Dairy Sci., 45, 1575.

Markiey R. A., Cason J. L., BAumGardt B. R., 1959. Effect of nitrogen fertilization or urea supplementation upon digestibility of grass hays. J. Dairy Sci., 42, I44-152.

Minson D.- J., Raymond W.- F., Harris C.- E., rg6o. Studies in the digestibility of herbage. VIII The digestibility of $S 23$ cocksfoot, $\$ 24$ rye grass and $\$ 37$ rye grass. J. Brit. Grassl. Soc., 15, 174-180.

Poulton B. R., Mac Donald G. J., VandernotT G. W., 1957. The effect of nitrogen fertilization on the nutritive value of orchard grass hay. J. Anim. Sci., 16, 463-466.

Poulton B. R., WoElfel C. G., I 963 . Effect of nitrogen fertilization upon the yield and digestibility of aftermath timothy forages fed to dairy heifers. J. Dairy Sci., 46, $46-49$.

Reid R. L., Clark B., Jung G. A., 1964. Studies with sudan grass. II. Nutritive evaluation by in vitro and in vivo methods. A gron. 5, 56, 537-542.

REID R. L., JuNG G.-A., I965. Influence of fertilizer treatment on the intake, digestibility and palatability of tall fescue hay. J. Anim. Sci., 24, 615-625.

Reid R. L., Jung G. A., Murray S. J., I966. Nitrogen fertilization in relation to the palatability and nutritive value of orchard grass. J. Anim. Sci., 25, 636-645.

Waite R., 1958. The water soluble carbohydrates of grasses. IV. The effect of different levels of fertilizer treatments. J. Sci. Food Agric., 9, 39 .

Washko J. B., MariotT L. F., I960. Yield and nutritive value of herbage as influenced by nitrogen fertilization in the northeastern United States. Proc. 8th Int. Grassl. Congr., I37-I4I.

Woelfel C. G., Poulton B. R., 1960. The nutritive value of timothy hay as affected by nitrogen fertilization. J. Anim. Sci., 19,695-699. 